\title{
High Damping Characteristics of an Elastomer Particle Damper
}

\author{
Marcelo Bustamante, Samir N. Y. Gerges and Erasmo F. Vergara \\ Federal University of Santa Catarina, Department of Mechanical Engineering, Laboratory of Vibration and Acous- \\ tics, Campus Universitario, P.B. 476, Trindade, Florianopolis, SC, CEP 88040-900, Brazil
}

\author{
Jorge P. Arenas \\ Institute of Acoustics, University Austral of Chile, PO Box 567, Valdivia, Chile
}

(Received 16 September 2014; accepted 14 December 2015)

\begin{abstract}
Research testing has led to the development of an Elastomer Particle Damper (EPD), which can add considerable damping to a structure by directing the vibration to a set of interacting elastomer particles through a rigid connection. This vibration treatment presents highly nonlinear behavior that is strongly dependent on both the vibration amplitude and frequency. Curves of damping loss factor (DLF) of an EPD system with vertical motion as a function of frequency and acceleration are reported herein. The results show that the elastomer particle damper has two distinct damping regions. The first region is related to the fluidization state of the particles, as described in the literature, obtained when the damper is subjected to vertical acceleration close to $1 \mathrm{~g}$ and frequencies below 50 $\mathrm{Hz}$. The second region presents high values of DLF to acceleration values lower than $1 \mathrm{~g}$, and the frequency range is dependent upon the stiffness of the particles. A high degree of effectiveness is achieved when the working frequency of the elastomer particle dampers is tuned to a natural frequency of a plate and when they are strategically located at points having large displacement. The performance of EPDs was compared with that of a commercial constrained layer damping installed in an aircraft floor panel. The EPDs achieved an acceleration level attenuation in the aircraft floor panel similar to that of the commercial constrained layer damping system.
\end{abstract}

\section{INTRODUCTION}

Traditional damping treatments use viscoelastic materials to convert strain energy into heat energy through the relative internal motion between molecules. Energy dissipation can be provided to a vibrating structure by a constrained damping layer in which a viscoelastic material is sandwiched between the structure to be damped and a stiff metal layer. Then, bending of the composite produces shear and the mechanical energy is dissipated in the middle layer as heat. These materials have been used quite successfully to address problems of noise and vibration control. ${ }^{1}$ However, the temperature sensitivity in polymer-damping processes is a major disadvantage. ${ }^{2}$ Another drawback is that the damper properties are strongly dependent on frequency and strain.

As an alternative, the use of particle dampers (PDs) can be an interesting solution. PDs are stiff enclosures containing a large number of either elastic or viscoelastic particles (e.g. sand, ball bearings, and elastomer balls) as shown in Fig. 1. Damping performance of PDs is usually not strongly temperature dependent and thus they can be used in harsh environments. Several studies have been carried out on PDs, mainly with metal spheres, providing modeling and experimental results. ${ }^{3-7}$ However, in this study, elastomer particles were used because the interaction between them is quieter, which is an important aspect in noise and vibration control.

PDs can be added to a structure in two ways: 1) by attaching an enclosure to an exterior surface or 2) by partially filling manufactured or pre-existing voids inside the structure with

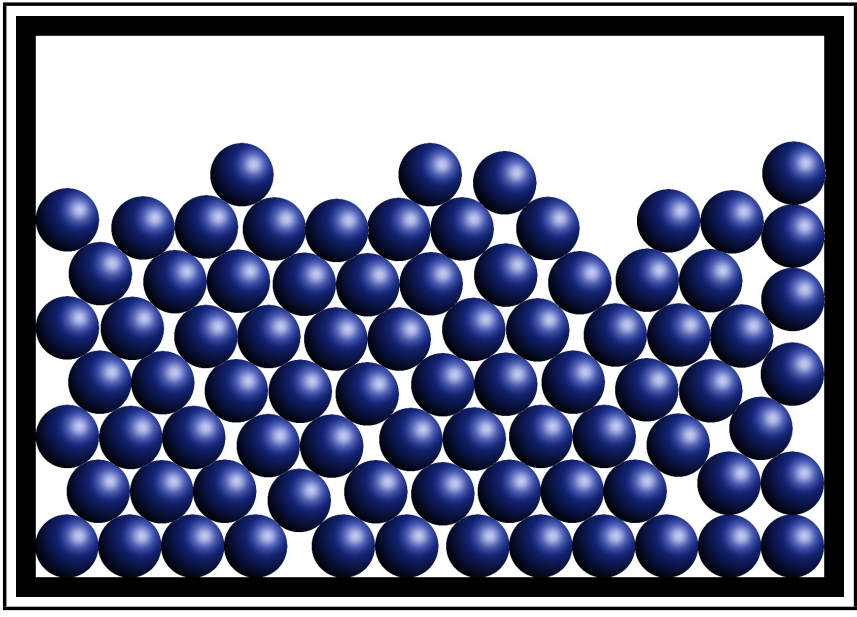

Figure 1. Schematic diagram of a particle damper.

particles.

The operating principle of PDs is based on energy dissipation through multiple inelastic collisions, interparticle friction, and friction between the particles and the walls of the container. The resulting system is highly nonlinear. Its damping capacity is greatly dependent on the level of acceleration which the container undergoes. There are a significant number of parameters affecting the damper performance. These include particle size, shape, number and density, the size and shape of the enclosure, and the properties that affect the particle-particle and particle-enclosure interactions, such as the coefficients of friction and restitution. ${ }^{3}$ 
PDs show different dynamic behaviors when the vibration level changes. When the vibration amplitudes are very low, the system is said to be in a "condensed state", where the particles are in contact with each other and moving together. When the amplitudes of vibration are high, it is said that the system is in a "fluidization state" where the particles move individually as fluid particles. ${ }^{8}$ During the transition between the two regimes, in some regions the particles are fluidized and in others they are still condensed. The presence of these two regimes and the transition from one to another is also dependent on the coefficient of restitution and the number of particles involved. Other authors have also considered the presence of a "gaseous state" of the particles as they adopt a chaotic and uncorrelated motion. ${ }^{9}$

The mass ratio between the particle damper and the primary system is also an important parameter to take into account. The size of a damping system with particle dampers is increased with an increasing in this mass ratio. ${ }^{10}$ Even with a low mass ratio, the particle dampers can be very effective in the attenuation of vibrations. ${ }^{11}$

In addition, particle size significantly affects the energy dissipation of the damper. ${ }^{12,13}$ A particle damper with very small particles dissipates energy mostly by friction. However, as the particle size increases the energy will be mainly dissipated through inelastic collisions. Furthermore, the efficiency of the damper is highly dependent on the coefficient of friction of the particles and not so much on the coefficient of restitution. ${ }^{14}$ In the first part of this article, the fluidization state of an EPD inserted in the free end of a beam fixed at its opposite end forming a single-degree-of-freedom (SDOF) system was investigated. Frequency Response Function (FRF) curves are obtained experimentally for different levels of excitation.

Subsequently, the study was expanded at frequencies of the EPD system using the Power Input Method (PIM). Using this method it was possible to raise the damping loss factor curves as a function of the acceleration and frequency experienced by the damper. The EPDs studied were applied for controlling a vibration mode of a steel plate and the vibration level of an aircraft floor plate.

Two types of elastomer particles were used and the curves for the stress versus percent elongation for these materials are shown in Fig. 2. These elastomers are referred to herein as elastomers A and B. Elastomer B is stiffer than elastomer A. The geometries of the elastomer particles are shown in Fig. 3. These geometries are very irregular and two different geometries of elastomer B were tested.

The gap formed by the free space between the container and the particles is an important parameter in relation to the damper efficiency. Results from several studies regarding the optimal gap have been published. ${ }^{10,11,15-19}$ However, for simplicity, PDs with roofless containers were considered in this study. Thus, no particle collisions occurred in the upper wall when the damper was subjected to accelerations greater than $1 \mathrm{~g}$ in the vertical direction.

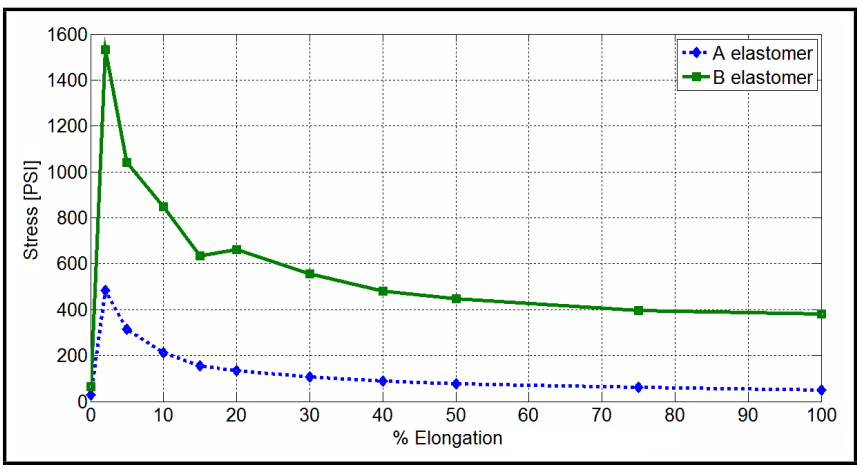

Figure 2. Curves for the stress versus percentage elongation of the elastomers used in the Elastomer Particle Damper (data provided by the manufacturer).

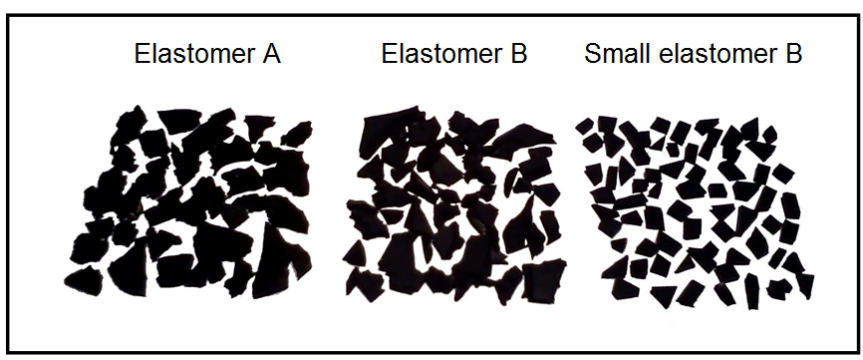

Figure 3. Geometry of elastomer particles used in the Elastomer Particle Damper.

\section{EXPERIMENTAL SET-UP MEASUREMENT SYSTEM}

\subsection{Inertance Curves of Beam-EPD SDOF System}

The experimental set-up shown in Fig. 4 was implemented for measuring the inertance in a SDOF cantilever beam system where the PD was located at the free end. The aluminum beam has a length of $280 \mathrm{~mm}$, a width of $38 \mathrm{~mm}$, and thickness of $3 \mathrm{~mm}$. The container was made of an acrylic rectangular box of inner dimensions $100 \mathrm{~mm}$ in length, $100 \mathrm{~mm}$ in width, and $50 \mathrm{~mm}$ in depth. The container was filled with $0.08 \mathrm{~kg}$ of elastomer A particles up to a height of $21.3 \mathrm{~mm}$. A vibration exciter was used to apply a force at the center of the beam. The applied force was measured using a force sensor inserted between the shaker and the beam. An accelerometer was located at the free end to measure the inertance FRF at this point relative to the force applied to the center of the beam. The vertical motion at the free end can be considered as an SDOF for the first mode shape of the beam and relatively high levels of acceleration are achieved.

A decrease in the length of the beam increased the natural frequency of the SDOF system. To achieve higher frequencies, it is necessary to clamp the two beam ends and place the particle damper at the center.

At its first natural frequency, the SDOF system was excited with a swept sine signal in order to obtain smoother curves and thus more precise damping loss factor calculations. A frequency bandwidth of $20 \mathrm{~Hz}$, with the center frequency close to the natural frequency of each SDOF system, was used. A set of inertance curves alternating the acceleration of the box from $-30 \mathrm{~dB}$ to $+10 \mathrm{~dB}$ (reference $1 \mathrm{~g}$ ) was obtained. 


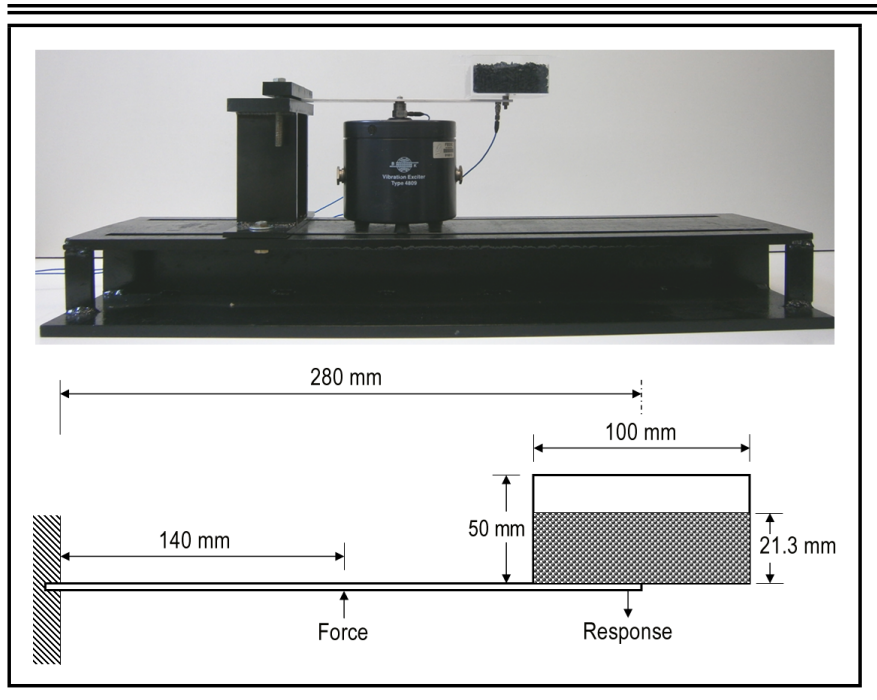

Figure 4. SDOF measurement system used to obtain the inertance FRF of the elastomer particle damper.

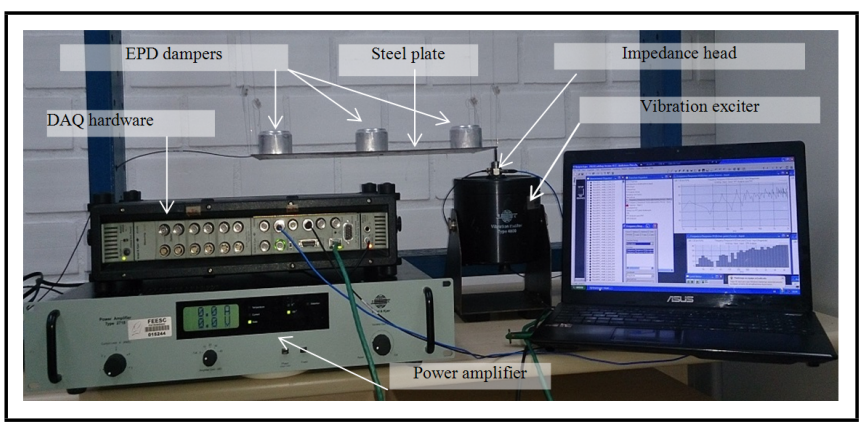

Figure 5. Measurement system used to obtain inertance curves of the steel plate with and without EPDs.

\subsection{Inertance Curves of a Steel Plate with and without EPDs}

The measurement system used to carry out the inertance measurements for a steel plate with and without EPDs is shown in Fig. 5. The steel plate, with dimensions $L_{x}=41.2 \mathrm{~cm}$, $L_{y}=21.02 \mathrm{~cm}$, and $L_{z}=0.21 \mathrm{~cm}$, and mass of $1.357 \mathrm{~kg}$ was discretized into 60 equally-spaced elements. The plate was hung from a metal structure using nylon cables. In this way, the plate was positioned horizontally and with free boundary conditions. A vibration exciter connected to a power amplifier was placed in the vertical direction at the right corner of the plate. A data acquisition system is controlled by software installed on a notebook. An impedance head was placed between the vibration exciter and the plate. An accelerometer was placed alternately at each of the sixty plate elements.

Three cylindrical EPDs were each attached to the plate with a total mass of $0.044 \mathrm{~kg}$. Therefore, the mass ratio (the ratio between the mass of the EPD and the mass of the primary system) was $0.044 \times 3 / 1.357=0.097$. The inertance measurement of the plate without dampers was carried out with only one acceleration input level of $-30 \mathrm{~dB}$, considering a linear system. In the case of the steel plate with dampers, the system is nonlinear, and the system was excited with different acceleration levels at inputs from $-42 \mathrm{~dB}$ to $+7 \mathrm{~dB}$ (reference $1 \mathrm{~g})$.

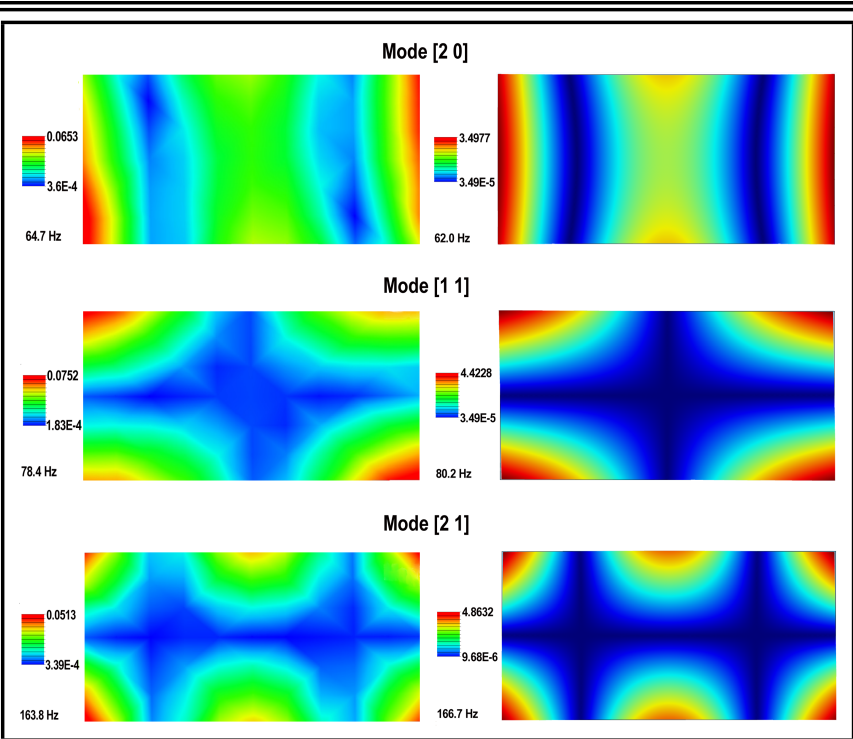

Figure 6. Experimental and simulated modal shapes of three first modes of a steel plate with dimensions $L_{x}=41.2 \mathrm{~cm}, L_{y}=21.02 \mathrm{~cm}$, and $L_{z}=0.21 \mathrm{~cm}$. Left: Experimental results. Right: Finite Element Analysis results.

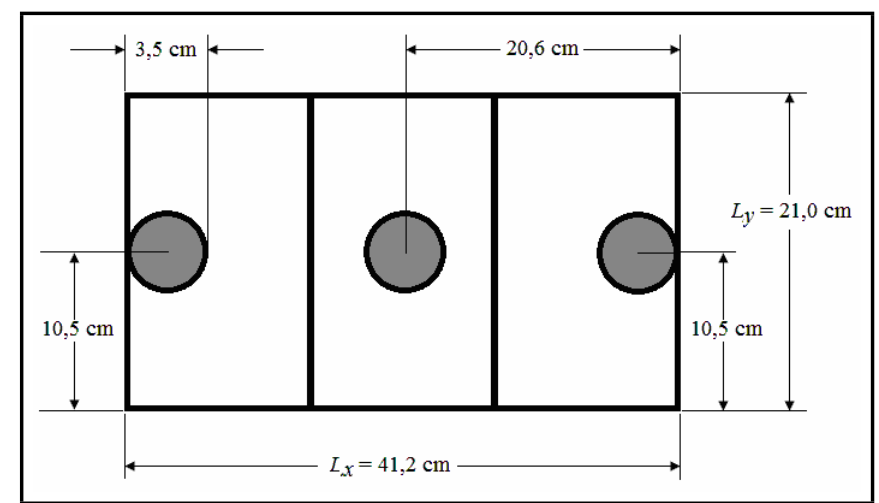

Figure 7. Nodal lines of the first mode and proper placement of dampers for damping the first mode of vibration.

\subsection{Defining the Quantity and Position of EPDs Needed for the First Mode of Vibration}

In order to determine the modal shapes of the steel plate and to define the EPD position, measurements and numerical simulations using a Finite Element (FE) approach were performed. The finite element modeling was performed using COMSOL Multyphysics 4.3. A plate geometry with dimensions $41.2 \times 21.02 \times 0.21 \mathrm{~cm}^{3}$ and free boundary conditions was defined. The plate was assumed isotropic with a Youngs modulus $E=210 \mathrm{GPa}$, density $\rho=7860 \mathrm{~kg} / \mathrm{m}^{3}$, and Poissons ratio $\nu=0.33$. A fine mesh of shell elements and COMSOL eigenfrequency solver were used in order to perform this modal analysis.

The three first mode shapes are shown in Fig. 6. The blue lines are nodal lines, and red areas indicate the maximum displacement. The mode shape of the first mode of the plate with free boundary conditions, defined as mode $[20]$, presents two nodal lines and three areas of displacement. Therefore, it is desirable to place at least one EPD inside each of these areas (see Fig. 7). 


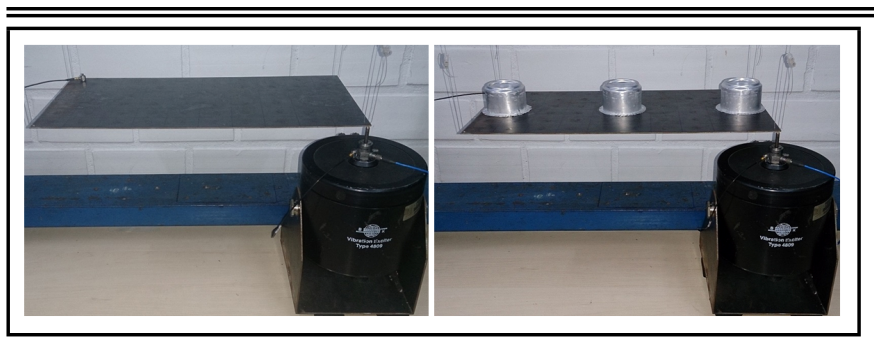

Figure 8. Steel plate with free boundary conditions. The vibration exciter was located at a corner of the plate and the accelerometer at the elements of the uniformly discretized plate. Left: Plate without dampers. Right: Plate with 3 EPDs. Dimensions are shown in Fig. 7.

Figure 8 shows a closer view of the uniformly discretized horizontal steel plate with and without EPDs. The vibration exciter appears in the lower right corner and the accelerometer is positioned at the opposite corner.

\subsection{Measurement Techniques Used}

\subsubsection{Half-power bandwidth method}

Half-power bandwidth method was used to measure the damping loss factor of the beam-EPD system and the vibration mode [2 0 ] of the steel plate with EPDs. This widely employed method uses an FRF of the structure, such as the inertance curve, and calculates the damping loss factor $\eta$ using the equation:

$$
\eta=\Delta \omega / \omega_{0}
$$

where $\Delta \omega$ is the bandwidth defined by the frequencies corresponding to the half-power point ( $-3 \mathrm{~dB}$ from peak value) and $\omega_{0}$ is the resonance frequency of the SDOF system. According to Blake, ${ }^{20}$ the damping of the system is defined with good approximation for $\eta$ values lesser than 0.2 .

In the plate, the driver point and response point were established within the areas of greater displacement of the vibration mode $[20]$. The driver point was located at an element situated on a corner, and the response point was located at an element at the center of the opposite side.

\subsubsection{Power input method}

Another method available to quantify the damping of a structure is the power input method (PIM). ${ }^{21,22}$ This method uses the following equation to determine the damping loss factor:

$$
\eta=\frac{\operatorname{Re}\left\{Y_{i i}(\omega)\right\}}{\sum_{j=1}^{N} m_{j} \omega\left|Y_{i j}(\omega)\right|^{2}} ;
$$

where $Y_{i i}$ is the point-driven mobility, $Y_{i j}$ is the transference mobility between the input point $i$ and the response point $j$, and $m_{j}$ is the mass of each of the $N$ elements of the discretized structure. For the application of the method the system must satisfy three key assumptions: ${ }^{23}$ (1) the replacement of strain energy with kinetic energy, (2) linearity of the system, i.e. the mobility is independent of amplitude, and (3) the structure can be suitably discretized so that the kinetic energy can adequately be determined with a modest number of observation points, each accurately representing the velocity of a discretized mass.

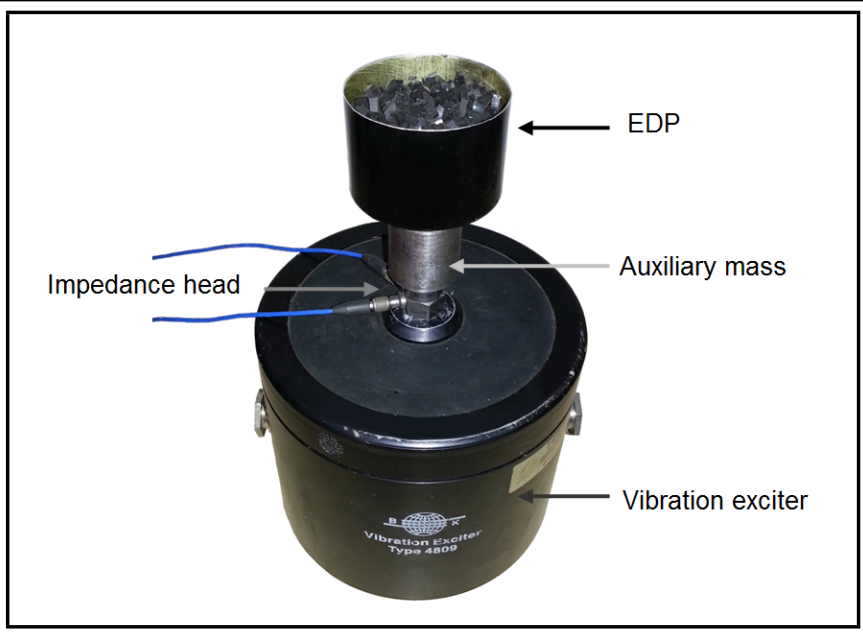

Figure 9. Experimental set-up used to measure the damping loss factor of an EPD as a function of acceleration and frequency.

As shown in Fig. 9, the EPD container was considered as a stiff mass, and it was connected directly to the vibration exciter. An impedance head was placed between the vibrator exciter and the EPD container. Thus, only the point-driven mobility curve $Y$ was necessary to measure. In this situation, Eq. (2) is written as

$$
\eta=\frac{\operatorname{Re}\{Y(\omega)\}}{M \omega|Y(\omega)|^{2}}
$$

where $M$ is the mass of the EPD container plus the particle mass.

This method has a significant advantage over other forms of damping loss factor measurement because it does not require the presence of a primary structure (as in the case of the beam-EPD system) and because measurements can be carried out at any acceleration level and frequency as desired. ${ }^{18}$ However, this relationship is approximate since the kinetic energy of particles is not considered.

PIM was also used applying Eq. (2) to obtain the damping loss factor for the steel plate with three EPDs considering the 60 elements of the uniformly discretized plate.

\subsubsection{Spatial-average response}

A commonly used measure of the plate surface vibration is the space-average value of the time-average squared vibration velocity defined by: ${ }^{24}$

$$
\left\langle\bar{v}^{2}\right\rangle=\frac{1}{S} \int_{S} \bar{v}^{2} d S
$$

where the upper bar denotes time-average and \langle\rangle denotes space-average. $S$ extends over the total vibrating surface of the plate, $v$ is the point velocity, and $\left\langle\bar{v}^{2}\right\rangle$ is known as the spatialaveraged mean-square velocity.

Similarly, the spatial-averaged mean-square mobility can be defined as

$$
\left\langle\bar{Y}^{2}\right\rangle=\frac{1}{S} \int_{S} \bar{Y}^{2} d S ;
$$

where $Y$ is the mobility function defined as $Y(\omega)=$ $v(\omega) / F(\omega)$. 
If a plate is discretized into $N$ elements, then Eq. (5) is

$$
\left\langle\bar{Y}^{2}\right\rangle=\frac{1}{S} \sum_{j=1}^{N} \bar{Y}_{i j}^{2} \Delta S_{j}
$$

where $i$ is the drive point where the force is applied. If the $N$ elements have the same dimensions and considering that $\bar{Y}^{2}=Y_{r m s}^{2}$, then the spatial-averaged mean-square inertance (or simply called spatial-averaged mobility) is given by:

$$
\left\langle\bar{Y}^{2}\right\rangle=\frac{1}{N} \sum_{j=1}^{N}\left(Y_{r m s}^{2}\right)_{i j} .
$$

The mobility curves were obtained exciting the system with a swept sine signal from $20 \mathrm{~Hz}$ to $2 \mathrm{kHz}$.

\subsection{Aircraft Floor Panel with EPDs}

In order to test the actual performance of the EPDs, the attenuation of the spatial-averaged response of an aircraft floor panel with 60 uniformly distributed EPDs on its surface and with a commercial constrained damping layer were compared. The aircraft panel is made of a honeycomb aluminum core and two epoxy/glass skin layers. The dimensions of the panel were $1.47 \mathrm{~m} \times 0.61 \mathrm{~m}$. The thickness and mass of the panel were $10.5 \mathrm{~mm}$ and $2.79 \mathrm{~kg}$, respectively. Each cylindrical EPD had a total mass of $0.0306 \mathrm{~kg}$. Therefore, the mass ratio was $0.0306 \times 60 / 2.79=0.66$. The mass of the damping layer was $1.917 \mathrm{~kg}$ and thus the mass ratio was 0.69 . The vibration treatments applied to the aircraft floor panel are shown in Fig. 10. Figure 11 shows the measurement system employed for this purpose. The vibration exciter was located at the bottom of the floor base, in the vertical direction, at two different points. The system was exited with white noise, and the response was measured at 8 randomly located points on the floor panel surface. Finally, the spatial-averaged response of the 8 points was calculated. A single acceleration-level curve was obtained allowing easy comparison of the performance of the two vibration control treatments applied to the floor panel.

\section{RESULTS AND DISCUSSION}

\subsection{Beam-EPD SDOF System}

\subsubsection{Fluidization state}

Recent research studies reported by the authors have led to the development of a type of particle damper consisting of elastomer particles of irregular geometry held within a container. ${ }^{10}$ Based on the work of Liu et al., this EPD was inserted into the SDOF experimental system shown in Fig. 4, and the FRF curves were obtained for different levels of excitation. ${ }^{3}$ White noise with a bandwidth of $50 \mathrm{~Hz}$ centered at $25 \mathrm{~Hz}$ was used as the excitation signal, and the results are shown in Fig. 12(a). A set of inertance FRF curves were obtained alternating the input power from $-30 \mathrm{~dB}$ to $+10 \mathrm{~dB}$ for an EPD with $0.08 \mathrm{~kg}$ of elastomer particles.

As noted above, this system is highly nonlinear. When the excitation level is low $(-30 \mathrm{~dB})$, the curves show a peak at a lower frequency compared with the corresponding curve for

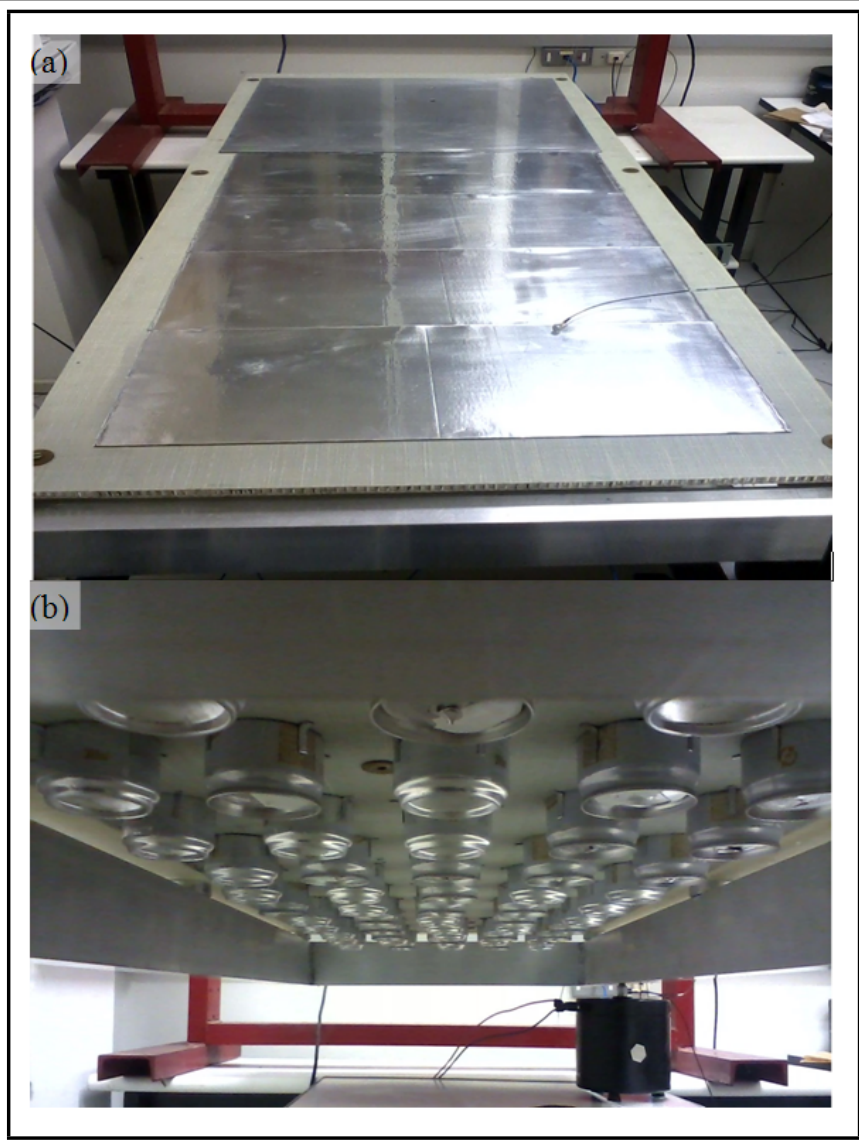

Figure 10. (a) Aircraft floor panel with a constrained layer damping system. (b) Aircraft floor panel with 60 elastomer particle dampers attached to the bottom and evenly spaced under the panel.

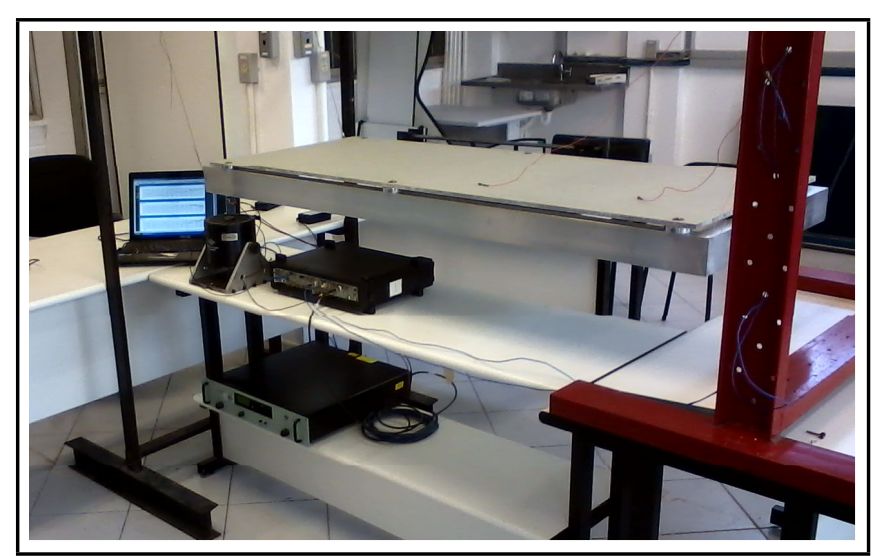

Figure 11. Mounting and measurement system used for testing the aircraft floor panel.

the empty box due to increased mass. As the level of excitation increases, the curves become wider and have a lower peak value which indicates an increase in the damping. Initially, the curves are smooth and later show fluctuations, which are due to the stick-slip friction mechanism, as explained by Papalou and Masri. ${ }^{4}$ As the level of excitation significantly increases, the frequency of the peak value on the FRF curve begins to shift toward that of the peak value on the curve for the empty box. This is because once the particles start to spend more time in the air, the effective mass decreases and there is a point at which the curve reaches the lowest peak value. This point was defined by the authors as the fluidization point of the particle 


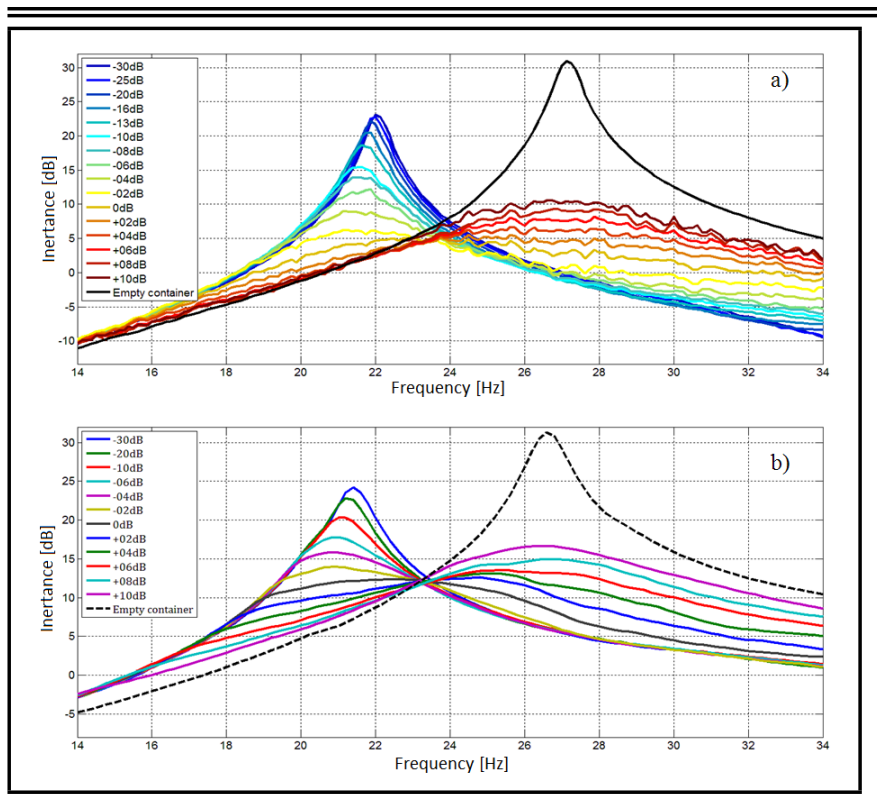

Figure 12. Inertance FRF curves for an elastomer particle damper in a SDOF system as a function of the power input level applied to the center of the beam. (a) System excited with white noise. (b) System excited with a swept sine signal.

damper. ${ }^{25}$ At this point, the attenuation of the peak value and damping achieved is the maximum possible for this damper. The frequency of the fluidization point is between the natural frequency of the system with the empty box and that for the box with particles. Note that the fluidization point is achieved when the EPD is subjected to vibration with acceleration close to $1 \mathrm{~g}$, that is, the acceleration needed to overcome gravity. Under this condition, the particles adopt the behavior of a moving fluid with random shape, and a high degree of damping is achieved through friction as well as the inelastic collision between the particles and the walls.

However, due to the high degree of irregularity associated with the FRF curves obtained for the EPD, which are shown in Fig. 12(a), it was difficult to accurately determine the center frequency and half-power band. This problem was solved by exciting the system with a swept sine signal, and smooth FRF curves were obtained. Fig. 12(b) shows an example of the FRF curves obtained with this type of signal. This is consistent with previously published results which indicated that the harmonic and random excitation will each cause the damper to behave in a different manner. ${ }^{4}$ Thus, the response of such dampers is also dependent on the type of excitation.

Figures 13 and 14 show the 3D plots of the inertance as a function of the acceleration and frequency of the SDOF system for the box without particles and the box containing $0.08 \mathrm{~kg}$ of elastomer particles, respectively. These figures facilitate an understanding of the fluidization phenomenon, which was explained above by comparing the response of the systems with and without particles. When particles are absent, the system is practically linear for the entire acceleration range studied. When the particles are placed in the box, greater attenuation by damping is achieved when the system is excited with a power input close to $0 \mathrm{~dB}$. Above this value, the frequency of the inertance peak values increases, tending toward the value corresponding to the empty box $(27 \mathrm{~Hz})$.

The results obtained in this previous study are encourag-

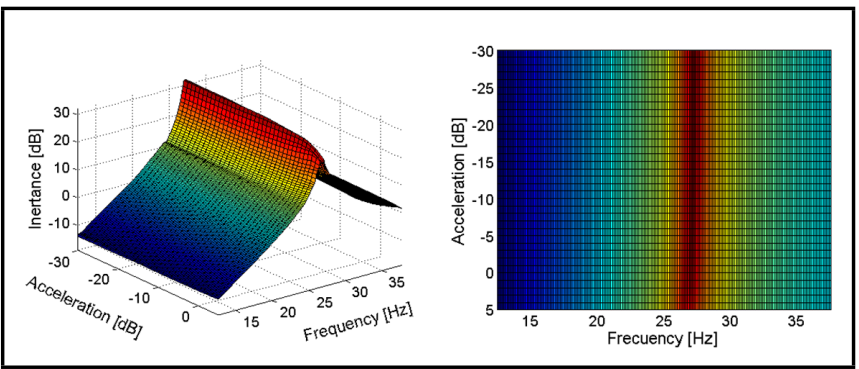

Figure 13. 3D plot of the inertance of the SDOF system with the box without particles as a function of the power input and frequency.

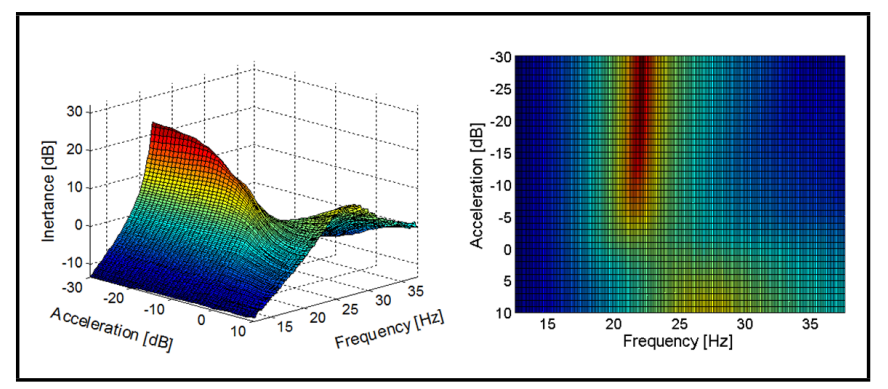

Figure 14. 3D plot of the inertance of the SDOF system with the box containing $0.08 \mathrm{~kg}$ of elastomer particles as a function of the power input and frequency.

ing. However, they were obtained for natural frequencies of the SDOF system between 20 and $30 \mathrm{~Hz}$. Thus, the dissipative behavior of the elastomer particle damper in a wider frequency range needs to be determined so that the applicability of this damper to noise and vibration problems can be established. Since this damping system is dependent on the acceleration to which it is subjected, this variable also needs to be studied. Thus, experimental curves for the damping as a function of the acceleration and frequency of an elastomer particle damper with $0.04 \mathrm{~kg}$ of particles were obtained and are reported herein.

\subsubsection{Damping loss factor as a function of accelera- tion and frequency of EPD system}

The damping loss factor of an EPD with $0.04 \mathrm{~kg}$ of elastomer particles was measured as a function of acceleration and frequency. The PIM was used considering Eq. (3). The results are shown in Figs. 15 to 18, each showing a 3D plot with two views. The left-hand image gives an isometric view and in the right-hand the graph is viewed from above (top view), showing the damping loss factor in the acceleration-frequency plane.

A damping region is present at low frequencies, starting at $20 \mathrm{~Hz}$, with acceleration value close to $0 \mathrm{~dB}$, namely around $1 \mathrm{~g}$ of acceleration, which is associated with the fluidization phenomenon observed in earlier studies. In addition to the damping region where fluidization occurred, it was found that this EPD presents another region of damping at an acceleration level lower than $0 \mathrm{~dB}$ (reference $1 \mathrm{~g}$ ). This is a positive feature because the EPD may also be used in structures subjected to relatively small acceleration levels.

Figure 15 shows the damping loss factor of the EPD system with $0.04 \mathrm{~kg}$ of elastomer A particles. We observed the region of highest damping between 30 and $40 \mathrm{~Hz}$. This region overlaps with the fluidization region.

Figure 16 shows the damping loss factor for the EPD sys- 


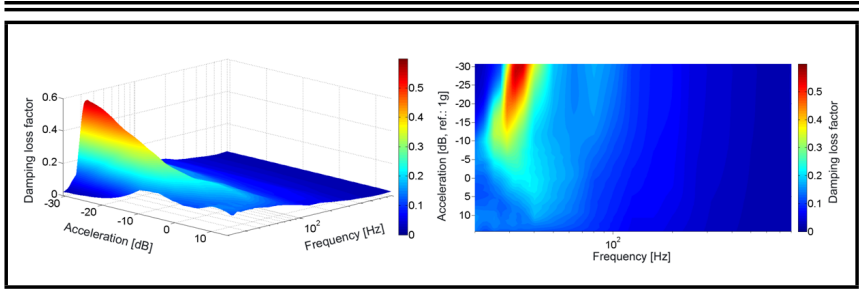

Figure 15. 3D plots for the damping loss factor of an EPD system with $0.04 \mathrm{~kg}$ of elastomer A particles as a function of acceleration and frequency.

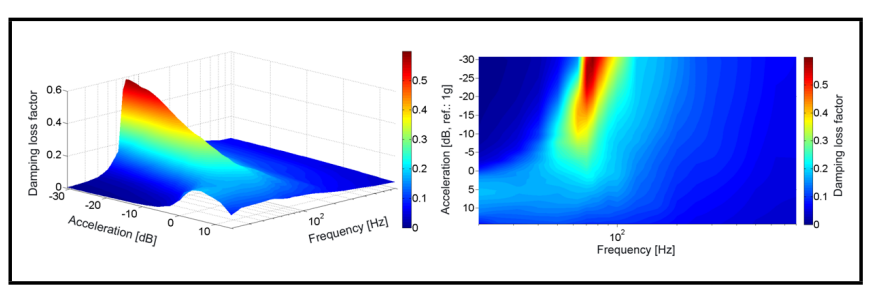

Figure 16. 3D plots for the damping loss factor of an EPD system with $0.04 \mathrm{~kg}$ of elastomer B particles as a function of acceleration and frequency.

tem with $0.04 \mathrm{~kg}$ of elastomer B particles. For this EPD, there is an increase in the frequency range of the maximum damping region to values between 60 and $90 \mathrm{~Hz}$. This is probably because elastomer B is stiffer than elastomer A. The region of fluidization is observed with greater clarity at low frequencies due to less overlapping with the other damping region.

Figure 17 shows the damping loss factor of the EPD system with $0.04 \mathrm{~kg}$ of small elastomer B particles. Since these particles are made of the same material as those related to the data in Fig. 16 but are of smaller size, they have a higher stiffness; therefore, the region of highest damping is shifted to frequencies in the range of 80 to $120 \mathrm{~Hz}$. This figure also clearly shows the fluidization region at around $1 \mathrm{~g}$ of acceleration.

Figure 18 shows the damping loss factor of the EPD system with a $0.04 \mathrm{~kg}$ rigid steel mass glued to the container (with no particles in the container). The damping obtained was not significant showing that in the previous cases damping was achieved due to the presence of the particles inside the box.

\subsection{Steel Plate with EPDs}

In order to control the vibration mode [2 0$]$ of the steel plate, three EPDs were applied. The working frequency of the EPDs was tuned to the natural frequency $f_{2,0}$ of the steel plate. The EPDs were strategically located as explained in Section 2.3.

These results are presented in two sections. Section 3.2.1 reports the measurements obtained with the half-power bandwidth method in order to analyze the attenuation achieved at the natural frequency $f_{2,0}$ by applying different levels of acceleration at the input. Section 3.2.2 reports the results obtained in the frequency band between $20 \mathrm{~Hz}$ and $2 \mathrm{kHz}$ for the spatial-

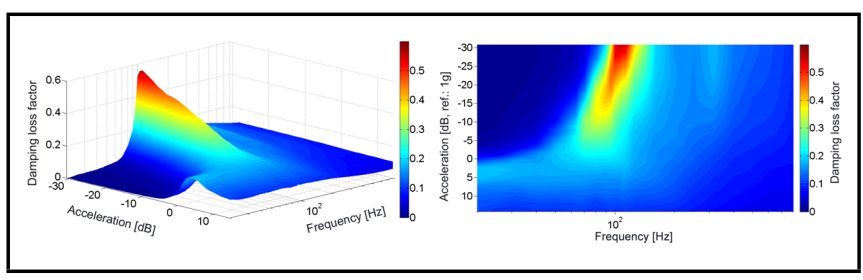

Figure 17. 3D plots for the damping loss factor of an EPD system with $0.04 \mathrm{~kg}$ of small elastomer B particles as a function of acceleration and frequency.

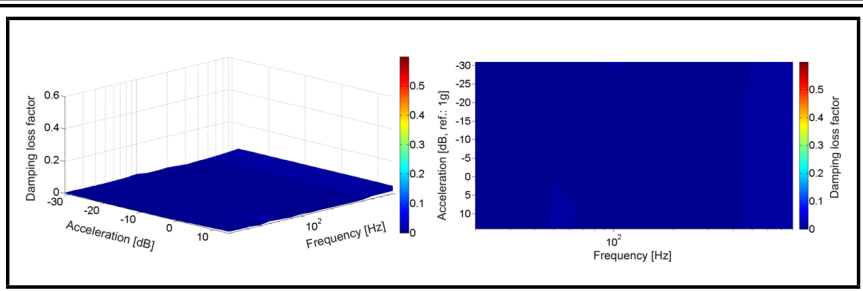

Figure 18. 3D plots for the damping loss factor of an EPD system with $0.04 \mathrm{~kg}$ of rigid mass (no particles in the container) as a function of acceleration and frequency.

averaged mobility and the damping loss factor of the steel plate with and without EPDs using the PIM method.

\subsubsection{Attenuation and damping at natural frequency $f_{2,0}$ of the steel plate}

Figure 19 shows the inertance curves centered at the natural frequency of the plate with and without dampers. The black curve corresponds to the plate without dampers and the other curves correspond to the plate with three EPDs, with different acceleration levels applied at the input. A large attenuation of the peak value is observed for each curve. It can be observed that, as expected, the lower the applied acceleration, the greater the damping peak value for the attenuation of the plate. This behavior is in contrast to that of the case when the EPD is not tuned to the natural frequency of the system, as is the case for the curves shown in Fig. 12. In this case, the curves exhibit greater attenuation when the EPD is subjected to accelerations close to $1 \mathrm{~g}$ and less attenuation when the acceleration decreases. In this situation, the damping region associated with the fluidization state of the particles prevails.

Figure 19b shows the same curves as that in Fig. 19a but in a $3 \mathrm{D}$ plot using data interpolation. The inertance curves are shown as a function of the frequency and acceleration experienced by the EPD. The color bar shows the inertance values (in $\mathrm{dB}$ ) associated with each color on the graph. The peak value of the inertance curve for the plate without dampers at natural frequency $f_{2,0}$ was $52.7 \mathrm{~dB}$. The greatest attenuation of the acceleration level was $31 \mathrm{~dB}$, obtained with the lowest level of acceleration at the damper of $-42 \mathrm{~dB}$.

The damping loss factor for each of the curves in Fig. 19a, obtained for the plate with three EPDs and without EPDs, was calculated using the half-power bandwidth method. The results are shown in Fig. 20. Given that the steel plate without dampers shows linear behavior, which means that the FRF curves do not vary with the amplitude of excitation, only one inertance measurement was taken and the calculated damping loss factor $\eta$ was 0.0032 . The damping loss factor of the plate with dampers was much higher for all levels of acceleration applied. The maximum value was 0.2 , obtained with the lowest level of input acceleration of $-42 \mathrm{~dB}$, in agreement with the attenuation results. The minimum value for $\eta$ was 0.07 , which was obtained with an input acceleration level of $-0.8 \mathrm{~dB}$.

\subsubsection{Attenuation and damping in the frequency range of $20 \mathrm{~Hz}$ to $2 \mathrm{kHz}$}

Mobility curves in the frequency range of $20 \mathrm{~Hz}$ to $2 \mathrm{kHz}$ were obtained for each of the 60 evenly spaced elements on the steel plate with and without the EPD. These curves were used 


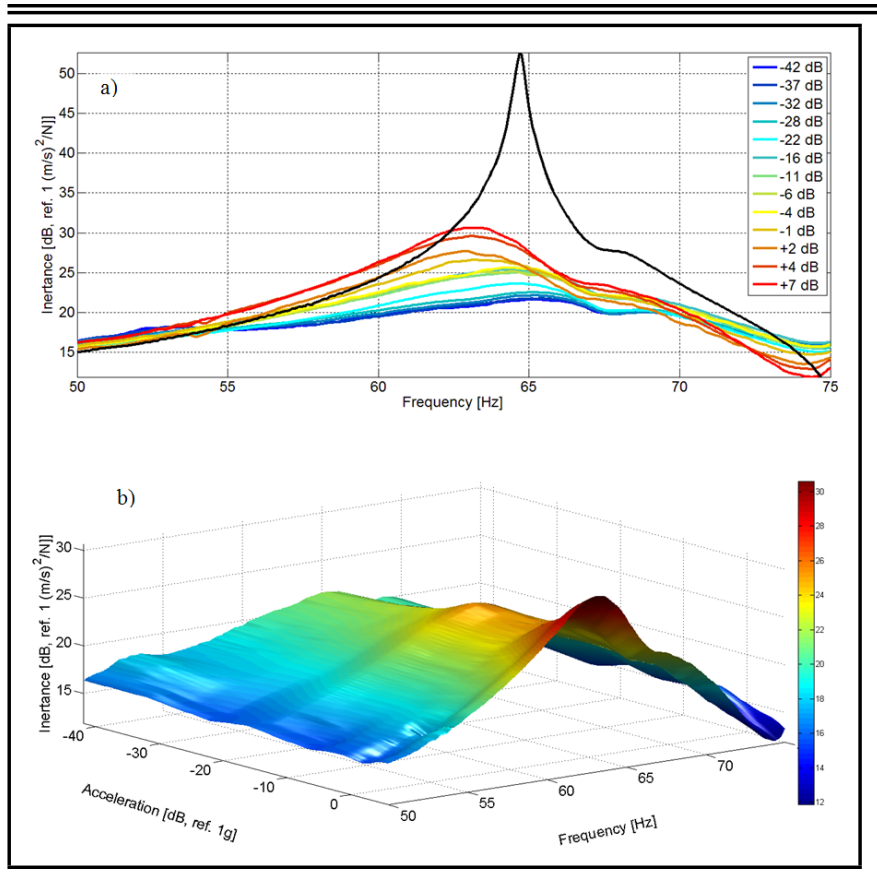

Figure 19. Inertance curves of the steel plate with three EPDs with different acceleration levels. (a) Inertance curves as a function of frequency. The black curve corresponds to the plate without EPDs. (b) The inertance curves presented in a) shown as a function of frequency and acceleration.

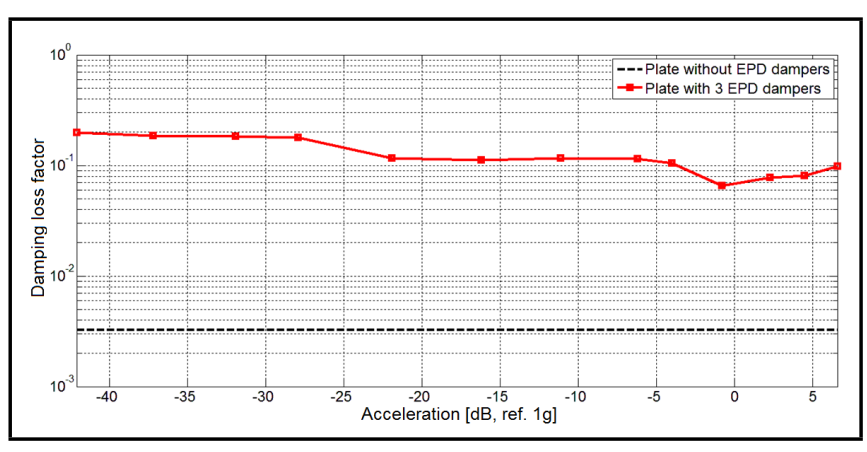

Figure 20. Damping loss factor of the steel plate with and without EPDs obtained with the half-power bandwidth method at natural frequency $f_{2,0}$.

to obtain the curves for the spatial-averaged mobility (shown in Fig. 21) and for the damping loss factor using the PIM method (shown in Fig. 22).

The peak corresponding to the natural frequency $f_{2,0}=$ $64 \mathrm{~Hz}$ of the steel plate without dampers was almost eliminated by the use of the EPDs as can be observed in Fig. 21. The peak attenuation value was close to $24 \mathrm{~dB}$. Above this natural frequency $f_{2,0}$, the EPDs still provide damping for the plate. At frequencies between $70 \mathrm{~Hz}$ and $2 \mathrm{kHz}$, it was observed that some peaks were greatly attenuated $(>15 \mathrm{~dB})$, others were slightly attenuated (around $5 \mathrm{~dB}$ ) and some were not attenuated. At the natural frequency $f_{1,1}=78 \mathrm{~Hz}$ the attenuation was small, and the peak value at the natural frequency $f_{2,1}=164 \mathrm{~Hz}$ was not attenuated. This is because in both cases, the dampers were located along the nodal lines of each mode where the displacement is zero; therefore, the vibration is not effectively transmitted to the dampers. The nodal lines can be seen in Fig. 6. Figure 22 shows the damping loss factor for the steel plate with three EPDs, obtained through the PIM method in the frequency range of $20 \mathrm{~Hz}$ to $2 \mathrm{kHz}$. A high damping peak centered at $60 \mathrm{~Hz}$, which defines the

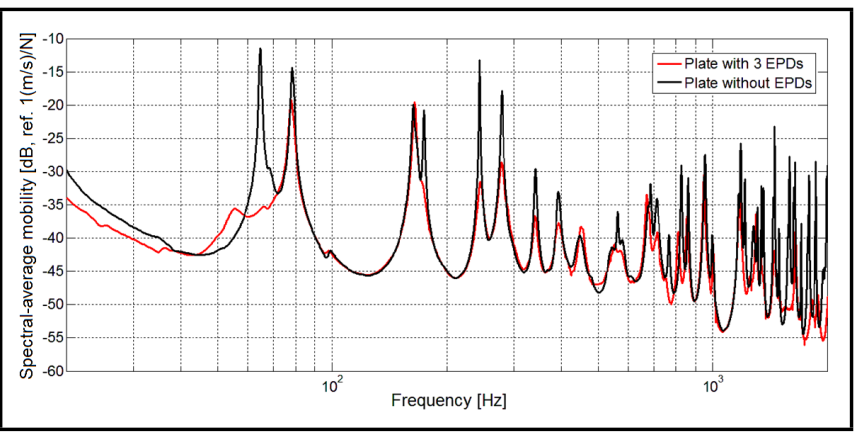

Figure 21. Spatial-averaged mobility curves of steel plate with and without EPDs.

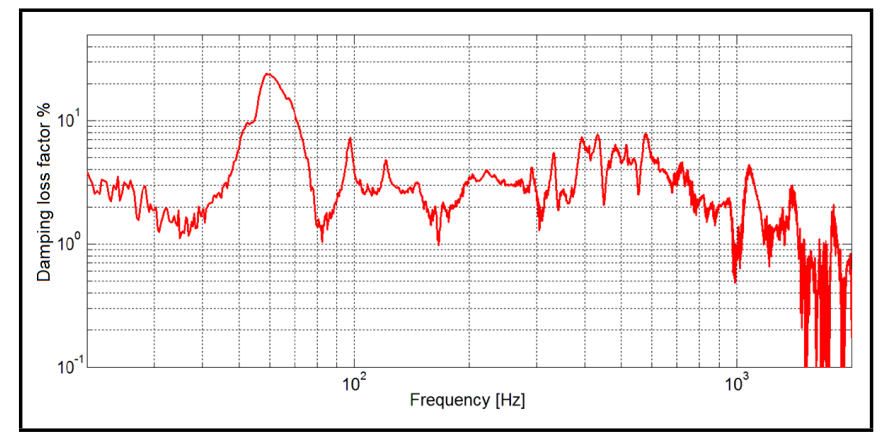

Figure 22. Damping loss factor of a steel plate with three EPDs obtained through the Power Input Method (PIM).

damper working frequency, is clearly observed. Furthermore, the dampers provided damping of over $2 \%$ across almost the entire frequency band analyzed, decreasing only above $700 \mathrm{~Hz}$, approximately. This damping curve is consistent with the attenuation obtained for the plate shown in Fig. 21, where the peak near $60 \mathrm{~Hz}$ was strongly attenuated and most other peaks were attenuated by the damping added to the plate.

\subsection{Aircraft Floor Panel with EPDs}

Figure 23 shows the spatial-averaged acceleration level of the aircraft floor panel without damping treatment. This curve is taken as a reference to calculate the vibration attenuation of the floor panel with a damping layer treatment.

Figure 24 shows the vibration attenuation of the spatialaveraged response of the aircraft floor panel with two types of vibration control treatments: a constrained damping layer and the 60 EPDs. It can be verified that the two treatments show similar performance, although higher attenuation is provided by the constrained layer at high frequencies (above $600 \mathrm{~Hz}$ ) and higher attenuation is provided by the EPDs at mid-range frequencies (60 to $300 \mathrm{~Hz}$ ).

\section{CONCLUSIONS}

An initial study on the dynamic behavior of a SDOF cantilever beam system with an EPD on the free end was carried out. A fluidization state was observed, as described in the literature. For the vertical displacement of the EPD, this state appears when the acceleration is close to $1 \mathrm{~g}$ and the particles adopt a motion similar to that of a fluid. The vibration energy dissipation mechanism is due to the friction and inelastic collisions between the particles themselves and between the 


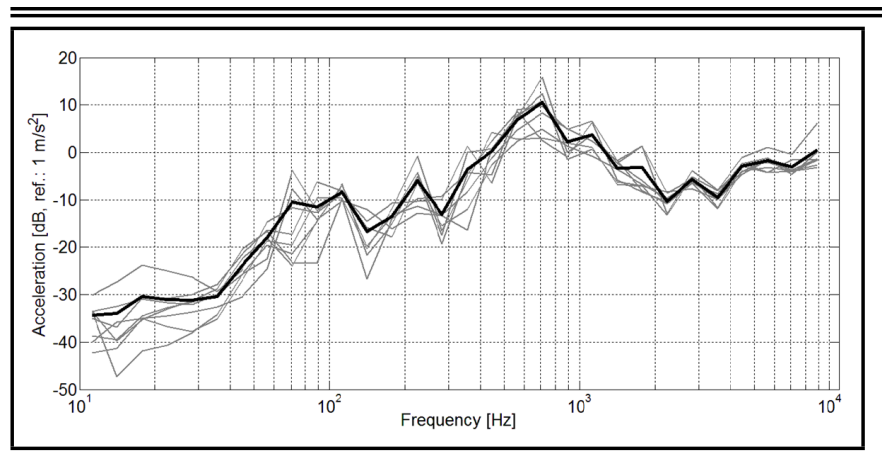

Figure 23. Acceleration level of the aircraft floor panel with stiff connection. Gray curves: acceleration level measured at each of eight randomly located points on the panel. Black curve: spatial-averaged acceleration level.

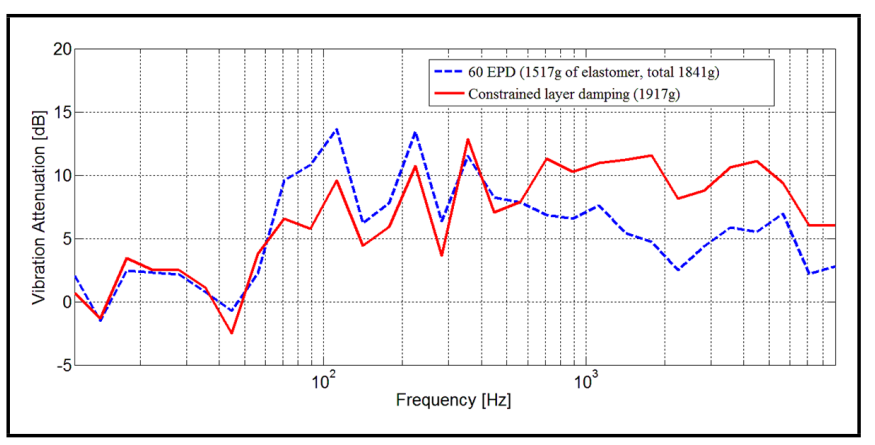

Figure 24. Attenuation of the spatial-averaged response of the aircraft floor panel with two damping vibration control techniques: 60 EPDs and a commercial constrained damping layer.

particles and the walls of the container. The inertance peak value decreases as the acceleration increases down to a minimum value before increasing again. When the minimum peak value on the curve is reached, the maximum possible damping is achieved by the system. Thus, it was appropriate to define the fluidization point of an EPD as the point at which this minimum inertance peak value occurs.

The study on the damping loss factor as a function of acceleration at a fixed frequency was expanded, and it was observed that the fluidization process is maintained for a few decades of frequency. In addition, another region where the particle dampers provided a high degree of damping was noticed. In this study, it was found that the frequency range was dependent upon the particle stiffness. Greater particle stiffness is associated with a higher center frequency of the band damping of the EPD.

These results open up a broader field of application, where the acceleration in the vertical direction of the particle damper is not limited to being close to $1 \mathrm{~g}$, but may be lower. However, this indicates the need to model the damping frequency as a function of parameters such as the stiffness, mass, and the coefficient of restitution of the particles. Discrete element models have been comprehensively applied to particle dampers and might represent a good alternative for modelling. ${ }^{12,13,26-30}$

The effectiveness of EPDs is greatly improved when the working frequency is tuned to a vibration mode of a plate and when the dampers are strategically located at points at which high displacement occurs. In particular, the three EPDs in this study, tuned to the vibration mode [2 0$]$ of a steel plate, were applied. The result was that the peak of the spatial-averaged mobility curve corresponding to this vibration mode was atten- uated by $24 \mathrm{~dB}$ and was almost eliminated. Above the working frequency of the EPD $(70 \mathrm{~Hz}$ to $2 \mathrm{kHz})$, the damper continues adding damping to the plate, achieving a damping loss factor of between 1 and $8 \%$. In this frequency range, the added damping achieves an attenuation peak of up to $15 \mathrm{~dB}$.

The performance of the EPDs was compared with that of a commercial constrained damping layer installed in an aircraft floor panel. The EPDs achieved an attenuation of the acceleration level on the aircraft floor panel similar to that of the commercial damping layer, with a somewhat better performance at mid-range frequencies and slightly poorer performance at high frequencies. These results are quite encouraging considering that elastomer particle dampers offer advantages such as easy construction, independence of temperature, and durability.

The work described in this article has been concerned with vertical excitation of the particle damper. Although the results presented here have demonstrated the effectiveness of the damper, further work should also address the effects on the damper performance of both the direction of excitation and the shape of the damper body.

\section{ACKNOWLEDGEMENTS}

The authors wish to acknowledge the representatives of ITT Enidine Inc. for partially funding this research project and to CNPq-Brazil for funding Ph.D. students and research staff.

\section{REFERENCES}

1 Xu, Z.-D., Zhu, J.-T., and Wang, D.-X. Analysis and optimisation of wind-induced vibration control for high-rise chimney structures. Int. J. Acoust. Vib. 19, 42-51, (2014).

2 Wetton, R. E. Design and measurement of polymeric materials for vibration absorption and control, Appl. Acoust., 11 (2), 77-97, (1978). http://dx.doi.org/10.1016/0003682X(78)90010-5

3 Liu, W., Tomlinson, G. R., and Rongong, J. A. The dynamic characterization of disk geometry particle dampers, J. Sound Vib., 280, 849861, (2005). http://dx.doi.org/10.1016/j.jsv.2003.12.047

4 Papalou, A. and Masri, S. F. Response of impact dampers with granular materials under random excitation, Earthquake Eng. Struct. Dyn., 25, 253267, (1996). http://dx.doi.org/10.1002/(sici)10969845(199603)25:3<253::aid-eqe553>3.3.co;2-w

5 Yang, M. Y. Development of master design curves for particle impact dampers, $\mathrm{PhD}$ diss., Dept. of Mechanical and Nuclear Engineering, The Pennsylvania State University, USA, (2003).

${ }^{6}$ Friend, R. D. and Kinra, V. K. Particle Impact Damping, J. Sound Vib., 233 (1), 93118, (2000). http://dx.doi.org/10.1006/jsvi.1999.2795

7 Darabi, B. and Rongong, J. A. Polymeric particle dampers under steady-state vertical vibrations, J. Sound Vib., 331, 33043316, (2012). http://dx.doi.org/10.1016/j.jsv.2012.03.005 
8 Duran, J. Sand, powders, and grains: An introduction to the physics of granular materials, Springer-Verlag, New York, (2000). http://dx.doi.org/10.1007/978-1-4612-0499-2

9 Saluea, C., Pschel, T., and Esipov, S. E. Dissipative properties of vibrated granular materials, Phys. Rev. E., 59 (4), 44224425, (1999). http://dx.doi.org/10.1103/PhysRevE.59.4422

10 Araki, Y., Yuhki, Y., Yokomichi, I., and Jinnouchi, Y. Impact Damper with Granular Materials: 3rd Report, Indicial Response, B. JSME, 28 (240), 12111217, (1985). http://dx.doi.org/10.1299/jsme1958.28.1211

11 Papalou, A. and Masri, S. F. An experimental investigation of particle dampers under harmonic excitation, J. Vib. Control, 4 (4), 361379, (1998). http://dx.doi.org/10.1177/107754639800400402

12 Chen, T., Mao, K., Huang, X., and Wang, M. Y. Dissipation mechanisms of nonobstructive particle damping using discrete element method, Proc. SPIE, 4331, 294301, (2001). http://dx.doi.org/10.1117/12.432713

13 Saeki, M. Impact damping with granular materials in a horizontally vibrating system, J. Sound Vib., 251 (1), 153161, (2002). http://dx.doi.org/10.1006/jsvi.2001.3985

14 Bai, X.-M., Shah, B., Keer, L. M., Wang, Q. J., and Snurr, R. Q. Particle dynamics simulations of a piston-based particle damper, Powder Technol., 189 (1), 115125, (2009). http://dx.doi.org/10.1016/j.powtec.2008.06.016

15 Bapat, C. N. and Sankar, S. Single unit impact damper in free and forced vibration, J. Sound Vib., 99 (1), 8594, (1985). http://dx.doi.org/10.1016/0022-460X(85)90446-8

16 Duncan, M. R., Wassgren, C. R., and Krousgrill, C. M. The damping performance of a single particle impact damper, J. Sound Vib., 286, 123144, (2005). http://dx.doi.org/10.1016/j.jsv.2004.09.028

17 Cempel, C. and Lotz, G. Efficiency of vibrational energy dissipation by moving shot, J. Struct. Eng., 119 (9), 26422652, (1993). http://dx.doi.org/10.1061/(ASCE)07339445(1993)119:9(2642)

18 Yang, M. Y., Lesieutre, G. A., Hambric, S. A., and Koopmann, G. H. Development of a design curve for particle impact dampers, Noise Control Eng., 53, 513, (2005). http://dx.doi.org/10.3397/1.2839240

19 Bannerman, M., Kollmer, J., and Sack, A. Movers and shakers: Granular damping in microgravity, Phys. Rev. E., 84, 011301, (2011). http://dx.doi.org/10.1103/PhysRevE.84.011301
20 Blake, R. E. Ch. 2: Basic vibration theory, Harris' Shock and Vibration Handbook, Allan G. Piersol and Thomas L. Paez Eds., McGraw-Hill, New York, (2010), 6th ed.

21 Carfagni, M. and Pierini, M. Determining the loss factor by the power input method (PIM), Part 1: Numerical investigation, J. Vib. Acoust. ASME, 121, 417421, (1999). http://dx.doi.org/10.1115/1.2893996

22 Carfagni, M. and Pierini, M. Determining the loss factor by the power input method (PIM), Part 2: Experimental investigation with impact hammer excitation, J. Vib. Acoust. ASME, 121, 422428, (1999). http://dx.doi.org/10.1115/1.2893997

23 Torvik, P. J. Ch. 35: Material and slip damping, Harris' Shock and Vibration Handbook, Allan G. Piersol and Thomas L. Paez Eds., McGraw-Hill, New York, (2010), 6th ed.

24 Fahy, F. J. and Gardonio, P. Sound and structural vibration-Radiation, transmission and response, Academic Press, Oxford, (2007), 2nd ed. http://dx.doi.org/10.3397/1.2741307

25 Bustamante, M., Gerges, S. N. Y., Cordioli, J., Martin, O., Weisbeck, J., and Ott, M. Experimental study on some parameters that affect the performance of an elastomer particle damper, Proc. ASA, Montreal, Canada, (2013). http://dx.doi.org/10.1121/1.4801066

26 Lu, Z., Masri, S. F., and Lu, X. Parametric studies of the performance of particle dampers under harmonic excitation, Struct. Control Hlth., 18, 7998, (2011). http://dx.doi.org/10.1002/stc.359

27 Snchez, M., Rosenthal, G., and Pugnaloni, L. A. Universal response of optimal granular damping devices, J. Sound Vib., 331, 43894394. (2012). http://dx.doi.org/10.1016/j.jsv.2012.05.001

28 Lu, Z., Lu, X., and Masri, S. F. Studies of the performance of particle dampers under dynamic loads, J. Sound Vib., 329, 54155433, (2010). http://dx.doi.org/10.1016/j.jsv.2010.06.027

29 Lu, Z., Lu, X., Lu, W., and Masri, S. F. Experimental studies of the effects of buffered particle dampers attached to a multi-degree-of freedom system under dynamic loads, J. Sound Vib., 331, 20072022 (2012). http://dx.doi.org/10.1016/j.jsv.2011.12.022

${ }^{30}$ Lu, Z., Lu, X., Lu, W., and Masri, S. F. Shaking table test of the effects of multi-unit particle dampers attached to an MDOF system under earthquake excitation, Earthq Eng. Struct. D., 41, 9871000, (2012). http://dx.doi.org/10.1002/eqe.1170 